\title{
ON AN EXTENSION OF GENERALIZED INCOMPLETE GAMMA FUNCTIONS WITH APPLICATIONS
}

\author{
M. ASLAM CHAUDHRY' ${ }^{1}$ and S. M. ZUBAIR ${ }^{2}$
}

(Received 19 July 1993; revised 22 February 1994)

\begin{abstract}
In this paper we have introduced extensions $\gamma_{\nu}(\alpha, x ; b)$ and $\Gamma_{\nu}(\alpha, x ; b)$ of the generalized Gamma functions $\gamma(\alpha, x ; b)$ and $\Gamma(\alpha, x ; b)$ considered recently by Chaudhry and Zubair. These extensions are found useful in the representations of the Laplace and $K$-transforms of a class of functions. We have also defined a generalization of the inverse Gaussian distribution. The cumulative and the reliability functions of the generalized inverse Gaussian distribution are expressed in terms of these functions. Some useful properties of the functions are also discussed.
\end{abstract}

\section{Introduction}

The inverse Gaussian density function

$$
g(t)=\left(\nu / 2 \pi t^{3}\right)^{1 / 2} e^{-v\left[(t-\mu)^{2} / 2 \mu t\right]}, \quad t, \mu, \nu>0,
$$

arises as the density of the first passage time of the Brownian motion with positive drift (see, [23], [24], [36], [44], [45], [50], [53], [58], [59], [71], [74]). The model has been used in the reliability theory and in the theory of demographic rates (see, [38], [44], [54], [68], [70], [73]). Good [33] proposed the generalized inverse Gaussian distribution

$$
h(t)=\frac{1}{I(\alpha ; a, b)} t^{\alpha-1} e^{-a t-b t^{-1}} \quad(t>0, a>0, b>0,-\infty<\alpha<\infty),
$$

which was used by Sichel ([68], [69]) to construct mixture of Poisson distributions. Barndorff-Nielsen ([10], [11]) used the generalized inverse Gaussian distribution (2) as a mixing distribution to obtain the generalized hyperbolic distribution as a

\footnotetext{
${ }^{1}$ Department of Mathematical Sciences

${ }^{2}$ Dept. of Mech. Eng., King Fahd University of Petroleum and Minerals, Dhahran, Saudi Arabia.

(C) Australian Mathematical Society, 1996, Serial-fee code 0334-2700/95
} 
mixture of normal distributions. Wise [74] used the model (2) in biomedicine and Marcus [44] used it as a unified stochastic model for the power laws in compartment analysis. Blaesild [14] discussed some probabilistic properties of (2). He computed the moments and cumulants and considered the shape of the density. Barndorff-Nielsen ([10], [11]) and Halgreen [36] showed that the density (2) is infinitely divisible and Halgreen [36] proved that (2) is self-decomposable (see also [40], [41]). Chhikara and Folks ([23], [24]) used the inverse Gaussian distribution (1) as a lifetime model and discussed its statistical applications.

Jorgensen [44] studied the distribution (2) systematically and discussed its applications in different fields like fractures of airconditioning equipment, traffic data, fracture toughness of MIG welds and repair time data (see also, [9], [11], [23], [38], [42], [54], [66]).

A natural generalization of the generalized inverse Gaussian model (2) is

$$
f(t)=C(\alpha ; a, b) t^{\alpha-1} e^{-a t} W_{0, v+\frac{1}{2}}(2 b / t) \quad(a>0, b \geq 0, t>0,-\infty<\alpha<\infty),
$$

where

$$
C=C(\alpha ; a, b)=\left(\int_{0}^{\infty} t^{\alpha-1} e^{-a t} W_{0, v+\frac{1}{2}}(2 b / t) d t\right)^{-1}
$$

is the normalizing constant and $W_{k, \mu}$ is one of the Whittaker functions [47]. It should be noted that for $v=0$ in (3) we get the generalized inverse Gaussian distribution (2). Several classical densities like Weibull, Gamma, Erlang, Exponential, Raleigh, and Chi-square can be derived from (2) by simple transformation of the variable $t$ or by specialization of the parameters $\alpha, a$ and $b$. Therefore all of these classical densities arising in diverse fields of applications are special cases of the model proposed in (3).

The study of the model (3) will provide a unified approach to the systematical analysis of the probability densities encountered in forestry, reliability theory and in demographic rates (see [19], [20], [54], [73]). The cumulative density function of the density (3) is

$$
F(x)=C \int_{0}^{x} t^{\alpha-1} e^{-a t} W_{0, v+\frac{1}{2}}(2 b / t) d t, \quad(x>0, b>0),
$$

and its reliability function is given by

$$
R(x)=1-F(x)
$$

The study of the functions $F(x)$ and $R(x)$ is important in statistics and in reliability theory. In particular, the systematic study of these functions will extend the usefulness of the generalized inverse Gaussian distributions in reliability and lifetesting situations with censored data. 
It should be noted that according to ([47], page 279) the Whittaker function $W_{0, v}$ can be expressed in terms of the Macdonald function to give

$$
K_{v}(z)=\sqrt{\frac{\pi}{2 z}} W_{0, \nu}(2 z)
$$

therefore, equations (3), (4) and (5) can be simplified in terms of the Macdonald function $K_{\nu}$ by using (7).

In this paper we introduce a pair of new functions

$$
\gamma_{\nu}(\alpha, x ; b)=\left(\frac{2 b}{\pi}\right)^{1 / 2} \int_{0}^{x} t^{\alpha-\frac{3}{2}} e^{-t} K_{v+\frac{1}{2}}(b / t) d t
$$

and

$$
\begin{gathered}
\Gamma_{\nu}(\alpha, x ; b)=\left(\frac{2 b}{\pi}\right)^{1 / 2} \int_{x}^{\infty} t^{\alpha-\frac{3}{2}} e^{-t} K_{\nu+\frac{1}{2}}(b / t) d t \\
(\operatorname{Re} x>0, \operatorname{Re} b>0,-\infty<\alpha<\infty)
\end{gathered}
$$

It follows from (5) - (9) that

$$
F(x)=C a^{-\alpha} \gamma_{\nu}(\alpha, a x ; a b)
$$

and

$$
R(x)=1-C a^{-\alpha} \Gamma_{v}(\alpha, a x ; a b) .
$$

It should be noted that the closed form solutions to a considerable number of problems in applied mathematics, astrophysics, nuclear physics, statistics and engineering can be expressed in terms of incomplete Gamma functions ([18], [21], [30], [35], [37], [43], [42], [47], [52], [57], [62], [59], [65], [75 - 77])

$$
\gamma(\alpha, x)=\int_{0}^{x} t^{\alpha-1} e^{-t} d t, \quad(\operatorname{Re} \alpha>0)
$$

and

$$
\Gamma(\alpha, x)=\int_{x}^{\infty} t^{\alpha-1} e^{-t} d t .
$$

These functions were investigated for real $x$ by Legendre ([48], [49]). The functional behavior of these functions and the decomposition formula

$$
\gamma(\alpha, x)+\Gamma(\alpha, x)=\Gamma(\alpha)
$$

were studied by Prym ([27], page 152). The older theory of the incomplete Gamma functions (12) - (13) and references to the literature are given by Nielsen ([55], [56]) 
and Böhmer [15]. Recently, Chaudhry and Zubair [22] have introduced generalized Gamma functions

$$
\begin{aligned}
& \gamma(\alpha, x ; b)=\int_{0}^{x} t^{\alpha-1} e^{-t-b t^{-1}} d t, \\
& \Gamma(\alpha, x ; b)=\int_{x}^{\infty} t^{\alpha-1} e^{-t-b t^{-1}} d t,
\end{aligned}
$$

found useful in the analytic study of a considerable number of heat conduction problems in a semi-infinite solid with time dependent boundary conditions ([22], [21], [75 - 77]) and in probability theory ([19], [20]). It should be noted that the substitution $v=0$ in (8) $-(9)$ leads to

$$
\gamma_{0}(\alpha, x ; b)=\gamma(\alpha, x ; b)
$$

and

$$
\Gamma_{0}(\alpha, x ; b)=\Gamma(\alpha, x ; b)
$$

Therefore the functions $\gamma_{\nu}(\alpha, x ; b)$ and $\Gamma_{\nu}(\alpha, x ; b)$ can be regarded as extensions of the generalized Gamma functions (15) - (16) found useful in statistics, applied mathematics and engineering.

In this paper we have studied important properties of these functions such as decomposition formula, special cases, differentiation formula, recurrence relations and Laplace transform representations. It is anticipated that the work presented in this paper will inspire scientists and engineers to find wide applications of these functions in several physical problems. It should be noted that for the most part the expressions used are analytic and hence retain their validity for the complex case because of the principle of analytic continuation. The proofs of some of the identities follow from the simple manipulations of the definitions (8) - (9); we have stated these identities as theorems for completeness.

\section{Main Results and Applications}

THEOREM 1. (Decomposition theorem)

$$
\begin{aligned}
& \gamma_{\nu}(\alpha, x ; b)+\Gamma_{\nu}(\alpha, x ; b) \\
& =2^{\alpha-2} \pi^{-1} b^{1 / 2} G_{04}^{40}\left(\frac{b^{2}}{16} \mid \frac{1}{2}\left(v+\frac{1}{2}\right),-\frac{1}{2}\left(v+\frac{1}{2}\right), \frac{1}{2}\left(\alpha+\frac{1}{2}\right), \frac{1}{2}\left(\alpha-\frac{1}{2}\right)\right), \\
& \quad(-\infty<\alpha<\infty, \operatorname{Re} b>0) .
\end{aligned}
$$

PROOF. Substituting $\mu=\frac{1}{2}$ in ([29], page 375(25)) and using the fact ([47], page 112)

$$
K_{1 / 2}(z)=\sqrt{\frac{\pi}{2 z}} e^{-z},
$$


we get

$$
\begin{aligned}
& \left(\frac{\pi}{2}\right)^{1 / 2} \int_{0}^{\infty} x^{\alpha-\frac{3}{2}} e^{-x} K_{\nu}(b / x) d x \\
& =2^{\alpha-3} G_{04}^{40}\left(\frac{b^{2}}{16} \mid \frac{v}{2},-\frac{v}{2}, \frac{1}{2}\left(\alpha+\frac{1}{2}\right), \frac{1}{2}\left(\alpha-\frac{1}{2}\right)\right) .
\end{aligned}
$$

Replacing $v$ by $v+\frac{1}{2}$ in (21) and simplifying we get

$$
\begin{aligned}
& \left(\frac{2 b}{\pi}\right)^{1 / 2} \int_{0}^{\infty} x^{\alpha-\frac{3}{2}} e^{-x} K_{v+\frac{1}{2}}(b / x) d x \\
& =2^{\alpha-2} \pi^{-1} b^{1 / 2} G_{04}^{40}\left(\frac{b^{2}}{16} \mid \frac{1}{2}\left(v+\frac{1}{2}\right),-\frac{1}{2}\left(v+\frac{1}{2}\right), \frac{1}{2}\left(\alpha+\frac{1}{2}\right), \frac{1}{2}\left(\alpha-\frac{1}{2}\right)\right) .
\end{aligned}
$$

From (8) - (9) and (22) we get the proof.

COROLlary.

$$
\gamma(\alpha, x ; b)+\Gamma(\alpha, x ; b)=2 b^{\alpha / 2} K_{\alpha}(2 \sqrt{b})
$$

PROOF. This follows from (18) when we substitute $v=0$ and use the fact ([28], page 217(18))

$$
G_{04}^{40}\left(z \mid-\frac{1}{4}, \frac{1}{4},\left(\frac{\alpha}{2}-\frac{1}{4}\right),\left(\frac{\alpha}{2}+\frac{1}{4}\right)\right)=4 \pi z^{(\alpha-1) / 4} K_{\alpha}\left(4 z^{1 / 4}\right) .
$$

COROLLARY.

$$
\gamma(\alpha, x)+\Gamma(\alpha, x)=\Gamma(\alpha), \quad(\operatorname{Re} \alpha>0)
$$

PROOF. This follows from (23) when we let $b \rightarrow 0^{+}$and use the fact ([47], page 136)

$$
\lim _{b \rightarrow 0} b^{\alpha} K_{\alpha}(b)=2^{\alpha-1} \Gamma(\alpha), \quad(\operatorname{Re} \alpha>0)
$$

REMARK.

$$
\Gamma_{-\nu-1}(\alpha, x ; b)=\Gamma_{\nu}(\alpha, x ; b)
$$

PROOF. This follows from the fact ([47], page 110) that $K_{\nu}(z)=K_{-\nu}(z)$. In particular, for $\nu=0$ in (27) we get

$$
\Gamma_{-1}(\alpha, x ; b)=\Gamma_{0}(\alpha, x ; b)=\Gamma(\alpha, x ; b) .
$$


THEOREM 2. (Recurrence relation)

$$
\begin{aligned}
& \Gamma_{v}(\alpha+1, x ; b) \\
& =(\alpha+\nu) \Gamma_{\nu}(\alpha, x ; b)+b \Gamma_{\nu-1}(\alpha-1, x ; b)+\left(\frac{2 b}{\pi}\right)^{1 / 2} e^{-x} x^{\alpha-\frac{1}{2}} K_{v+\frac{1}{2}}(b / x), \\
& \quad(\operatorname{Re} b>0,-\infty<\alpha<\infty) .
\end{aligned}
$$

PROOF. According to ([34], page 970(8.486)(12)), we have

$$
\frac{d}{d t}\left[K_{v+\frac{1}{2}}(b / t)\right]=\frac{b}{t^{2}} K_{\nu-\frac{1}{2}}(b / t)+\left(\nu+\frac{1}{2}\right) t^{-1} K_{\nu+\frac{1}{2}}(b / t) .
$$

Differentiating $t^{\alpha-\frac{1}{2}} e^{-t} K_{v+\frac{1}{2}}(b / t)$ with respect to $t$ and using (30), we get

$$
\begin{aligned}
& \frac{d}{d t}\left[t^{\alpha-\frac{1}{2}} e^{-t} K_{v+\frac{1}{2}}(b / t)\right] \\
& =(\alpha+\nu) t^{\alpha-\frac{3}{2}} e^{-t} K_{\nu+\frac{1}{2}}(b / t)+b t^{\alpha-\frac{5}{2}} e^{-t} K_{\nu-\frac{1}{2}}(b / t)-t^{\alpha-\frac{1}{2}} e^{-t} K_{v+\frac{1}{2}}(b / t) .
\end{aligned}
$$

Multiplying both sides in (31) by $\left(\frac{2 b}{\pi}\right)^{1 / 2}$ and integrating from $x$ to $\infty$ and using (9), we get

$$
\begin{aligned}
& 0-\left(\frac{2 b}{\pi}\right)^{1 / 2} x^{\alpha-\frac{1}{2}} e^{-x} K_{\nu+\frac{1}{2}}(b / x) \\
& =(\alpha+\nu) \Gamma_{\nu}(\alpha, x ; b)+b \Gamma_{\nu-1}(\alpha-1, x ; b)-\Gamma_{\nu}(\alpha+1, x ; b) .
\end{aligned}
$$

A rearrangement of the terms in (32) yields the proof of the theorem.

COROLlaRY. (See [22])

$$
\Gamma(\alpha+1, x ; b)=\alpha \Gamma(\alpha, x ; b)+b \Gamma(\alpha-1, x ; b)+x^{\alpha} e^{-x-b x^{-1}} .
$$

PROOF. This follows from (29) when we substitute $v=0$ and use equations (20) and (28). In particular substituting $b=0$ in (33) we get the recurrence relation ([34], page 942(8.356))

$$
\Gamma(\alpha+1, x)=\alpha \Gamma(\alpha, x)+x^{\alpha} e^{-x}
$$

for the classical incomplete Gamma function.

THEOREM 3. (Laplace transform representations) Let

$$
H(\tau)=\left\{\begin{array}{l}
1, \tau>0 \\
0, \tau<0
\end{array}\right.
$$


be the Heaviside unit step function and $L$ be the Laplace transform operator. Then

$$
L\left\{t^{\alpha-\frac{3}{2}} K_{\nu+\frac{1}{2}}(b / t) H(t-x) ; s\right\}=\left(\frac{\pi}{2 b}\right)^{1 / 2} s^{-\alpha} \Gamma_{\nu}(\alpha, s x ; s b)
$$

and

$$
\begin{gathered}
L\left\{t^{\alpha-\frac{3}{2}} K_{\nu+\frac{1}{2}}(b / t) H(x-t) H(t) ; s\right\}=\left(\frac{\pi}{2 b}\right)^{1 / 2} s^{-\alpha} \gamma_{\nu}(\alpha, s x ; s b), \\
(x \geq 0, \operatorname{Re} b>0,-\infty<\alpha<\infty)
\end{gathered}
$$

PROOF. By definition of the Laplace transformation we have

$$
\begin{aligned}
& L\left\{t^{\alpha-\frac{3}{2}} K_{v+\frac{1}{2}}(b / t) H(t-x) ; s\right\} \\
& =\int_{0}^{\infty} t^{\alpha-\frac{3}{2}} K_{v+\frac{1}{2}}(b / t) e^{-s t} H(t-x) d t, \\
& =\int_{x}^{\infty} t^{\alpha-\frac{3}{2}} K_{v+\frac{1}{2}}(b / t) e^{-s t} d t .
\end{aligned}
$$

Substituting $t=\tau / s, d t=d \tau / s$, we get

$$
\int_{x}^{\infty} t^{\alpha-\frac{3}{2}} K_{v+\frac{1}{2}}(b / t) e^{-s t} d t=s^{-\alpha+\frac{1}{2}} \int_{s x}^{\infty} \tau^{\alpha-\frac{3}{2}} e^{-\tau} K_{v+\frac{1}{2}}(b s / \tau) d \tau
$$

From (9), (37) and (38), we get the proof of (35). The proof of (36) follows similarly.

\section{COROLLARY.}

$$
\begin{aligned}
& L\left\{t^{-1 / 2} e^{-b / t} H(t-x) ; s\right\} \\
& =\frac{\sqrt{\pi}}{2 \sqrt{s}}\left[e^{-2 \sqrt{b s}} \operatorname{Erfc}\left(\sqrt{s x}-\sqrt{\frac{b}{x}}\right)+e^{2 \sqrt{b s}} \operatorname{Erfc}\left(\sqrt{s x}+\sqrt{\frac{b}{x}}\right)\right], \\
& L\left\{t^{-3 / 2} e^{-b / t} H(t-x) ; s\right\} \\
& =\frac{\sqrt{\pi}}{2 \sqrt{b}}\left[e^{-2 \sqrt{b s}} \operatorname{Erfc}\left(\sqrt{s x}-\sqrt{\frac{b}{x}}\right)-e^{2 \sqrt{b s}} \operatorname{Erfc}\left(\sqrt{s x}+\sqrt{\frac{b}{x}}\right)\right] .
\end{aligned}
$$

Proof. These identities follow from (35) when we substitute $v=0, \alpha= \pm 1 / 2$ and use the fact [22]

$$
\Gamma\left(\frac{1}{2}, x ; b\right)=\frac{\sqrt{\pi}}{2}\left[e^{-2 \sqrt{b}} \operatorname{Erfc}\left(\sqrt{x}-\sqrt{\frac{b}{x}}\right)+e^{2 \sqrt{b}} \operatorname{Erfc}\left(\sqrt{x}+\sqrt{\frac{b}{x}}\right)\right]
$$


and

$$
\sqrt{b} \Gamma\left(-\frac{1}{2}, x ; b\right)=\frac{\sqrt{\pi}}{2}\left[e^{-2 \sqrt{b}} \operatorname{Erfc}\left(\sqrt{x}-\sqrt{\frac{b}{x}}\right)-e^{2 \sqrt{b}} \operatorname{Erfc}\left(\sqrt{x}+\sqrt{\frac{b}{x}}\right)\right] .
$$

It should be noted that the identities (39) and (40) are sharp and do not seem to be known in the literature. In particular, when we take $b=0$ in (39) we get (see [28], page $135(15)$ )

$$
L\left\{t^{-1 / 2} H(t-x) ; s\right\}=\sqrt{\pi} s^{-1 / 2} \operatorname{Erfc}(\sqrt{s x}), \quad x>0, s>0 .
$$

THEOREM 4. (Evaluation of $\Gamma_{\nu}(\alpha, x ; b)$ for integral values of $\left.\nu\right)$ Let $\Gamma(\alpha, x ; b)$ be the generalized gamma function as defined by (9). Then

$$
\Gamma_{n}(\alpha, x ; b)=\sum_{m=0}^{n} \frac{(2 b)^{-m}}{m !} \frac{\Gamma(n+m+1)}{\Gamma(n-m+1)} \Gamma(\alpha+m, x ; b) \quad(n \in\{0,1,2,3, \ldots\}) .
$$

PROOF. This follows from the representation (9) and from the fact ([27], page 10) that

$$
K_{n+\frac{1}{2}}(z)=\sqrt{\frac{\pi}{2 z}} e^{-z} \sum_{m=0}^{n} \frac{(2 z)^{-m}}{m !} \frac{\Gamma(n+m+1)}{\Gamma(n-m+1)} .
$$

REMARK. Since the generalized gamma function $\Gamma\left(\frac{1}{2}+m, x ; b\right), m \in\{0, \pm 1, \pm 2$, $\pm 3, \ldots\}$, can be simplified in terms of error functions [22], it follows from (44) that for $\alpha=r+\frac{1}{2}, r \in\{0, \pm 1, \pm 2, \pm 3, \ldots\}$, the function $\Gamma_{n}\left(r+\frac{1}{2}, x ; b\right)$ is expressible in terms of error functions. Moreover, according to (27), $\Gamma_{-n-1}(\alpha, x ; b)=\Gamma_{n}(\alpha, x ; b)$, therefore, $\Gamma_{n}\left(r+\frac{1}{2}, x ; b\right)$ should be simplified in terms of error functions for all $r, n \in\{0, \pm 1, \pm 2, \pm 3, \ldots\}$.

THEOREM 5. (Parametric differentiation)

$$
\frac{\partial}{\partial b}\left(\Gamma_{\nu}(\alpha, x ; b)\right)=-\frac{1}{b}\left[\nu \Gamma_{\nu}(\alpha, x ; b)+b \Gamma_{\nu-1}(\alpha-1, x ; b)\right]
$$

PROOF. Differentiating both sides of (9) with respect to the parameter $b$ we get

$$
\begin{aligned}
\frac{\partial}{\partial b}\left(\Gamma_{v}(\alpha, x ; b)=\right. & \frac{1}{2 b}\left(\frac{2 b}{\pi}\right)^{1 / 2} \int_{x}^{\infty} t^{\alpha-\frac{3}{2}} e^{-t} K_{v+\frac{1}{2}}(b / t) d t \\
& +\left(\frac{2 b}{\pi}\right)^{1 / 2} \int_{x}^{\infty} t^{\alpha-\frac{3}{2}} e^{-t} \frac{\partial}{\partial b}\left[K_{v+\frac{1}{2}}(b / t)\right] d t
\end{aligned}
$$


It should be noted that the process of differentiation under the integral sign in (46) is justified ([16], pages 427-448). Using the differentiation formula ([32, page 970(8.486)(12)) for the Macdonald function, we get

$$
\begin{aligned}
& \frac{\partial}{\partial b}\left(\Gamma_{v}(\alpha, x ; b)\right) \\
& =\frac{1}{2 b}\left(\frac{2 b}{\pi}\right)^{1 / 2} \int_{x}^{\infty} t^{\alpha-\frac{3}{2}} e^{-t} K_{v+\frac{1}{2}}(b / t) d t-\frac{1}{b}\left(\frac{2 b}{\pi}\right)^{1 / 2} \\
& \quad-\int_{x}^{\infty} t^{\alpha-\frac{3}{2}} e^{-t}\left\{\left(\nu+\frac{1}{2}\right) K_{v+\frac{1}{2}}+\frac{b}{t} K_{v-\frac{1}{2}}(b / t)\right\} d t \\
& =\frac{1}{2 b} \Gamma_{\nu}(\alpha, x ; b)-\frac{1}{b}\left\{\left(v+\frac{1}{2}\right) \Gamma_{v}(\alpha, x ; b)+b \Gamma_{\nu-1}(\alpha-1, x ; b)\right\} .
\end{aligned}
$$

The simplification in (47) yields the proof of (45). In particular for $v=0$ in (45), we get the differentiation formula [22]

$$
\frac{\partial}{\partial b}(\Gamma(\alpha, x ; b)=-\Gamma(\alpha-1, x ; b)
$$

for the generalized incomplete gamma function.

THEOREM 6. ( $K$-transform Representation) Let $R_{v}$ be the $K$-transform operator as defined by ([23], page 125)

$$
R_{v}[f(t) ; b]=\int_{0}^{\infty} f(t) K_{v}(b t)(b t)^{1 / 2} d t .
$$

Then

$$
R_{v}\left[t^{-\alpha-1} e^{-1 / t} H(x-t) H(t) ; b\right]=\left(\frac{\pi}{2}\right)^{1 / 2} \Gamma_{\nu-\frac{1}{2}}\left(\alpha, \frac{1}{x} ; b\right)
$$

and

$$
R_{v}\left[t^{-\alpha-1} e^{-1 / t} H(t-x) ; b\right]=\left(\frac{\pi}{2}\right)^{1 / 2} \gamma_{\nu-\frac{1}{2}}\left(\alpha, \frac{1}{x} ; b\right)
$$

ProOF. Replacing $x$ by $1 / x$ and $v$ by $v-\frac{1}{2}$ in (9), we get

$$
\Gamma_{\nu-\frac{1}{2}}\left(\alpha, \frac{1}{x} ; b\right)=\left(\frac{2 b}{\pi}\right)^{1 / 2} \int_{1 / x}^{\infty} \xi^{\alpha-\frac{3}{2}} e^{-\xi} K_{\nu}(b / \xi) d \xi .
$$

Substituting $\xi=1 / t, d \xi=-d t / t^{2}$ in (50), we get

$$
\int_{1 / x}^{\infty} \xi^{\alpha-\frac{3}{2}} e^{-\xi} K_{\nu}(b / \xi) d \xi=\int_{0}^{x} t^{-\alpha-1} e^{-1 / t} K_{\nu}(b t) t^{1 / 2} d t .
$$


From (50) and (51), we get

$$
\begin{aligned}
\Gamma_{\nu-\frac{1}{2}}\left(\alpha, \frac{1}{x} ; b\right) & =\left(\frac{2}{\pi}\right)^{1 / 2} \int_{0}^{x} t^{-\alpha-1} e^{-1 / t}\left[K_{\nu}(b t)(b t)^{1 / 2}\right] d t \\
& =\left(\frac{2}{\pi}\right)^{1 / 2} \int_{0}^{\infty} t^{-\alpha-1} e^{-1 / t} H(x-t) H(t)\left[K_{\nu}(b t)(b t)^{1 / 2}\right] d t
\end{aligned}
$$

From (51) and (52), we get the proof of (48).

The proof of (49) follows similarly. It should be noted that several special cases of (48) - (49) can be listed.

REMARK. The equation (44) gives the relationship between $\Gamma_{\nu}(\alpha, x ; b)$ and $\Gamma(\alpha, x ; b)$ for integral values of $\nu$. For non-integral values of $\nu$ we have not been able to express $\Gamma_{\nu}(\alpha, x ; b)$ in terms of other tabulated classical functions and this remains an open problem. However, we have the following important result.

THEOREM 7. (Integration with respect to the parameter $v$ )

$$
\begin{aligned}
& \Gamma\left(\alpha+\frac{1}{2}, \frac{1}{x} ; b\right)=\left(\frac{2}{\pi}\right)^{1 / 2} b^{\alpha} \int_{0}^{\infty} \gamma_{-\frac{1}{2}+i \nu}(-\alpha, x ; b) d \nu, \\
& \gamma\left(\alpha+\frac{1}{2}, \frac{1}{x} ; b\right)=\left(\frac{2}{\pi}\right)^{1 / 2} b^{\alpha} \int_{0}^{\infty} \Gamma_{-\frac{1}{2}+i \nu}(-\alpha, x ; b) d \nu .
\end{aligned}
$$

PROOF. According to (8) we have

$$
\gamma_{-\frac{1}{2}+i \nu}(-\alpha, x ; b)=\left(\frac{2 b}{\pi}\right)^{1 / 2} \int_{0}^{x} t^{-\alpha-\frac{3}{2}} e^{-t} K_{i v}(b / t) d t
$$

Substituting $t=1 / \tau, d t=-d \tau / \tau^{2}$ we get

$$
\gamma_{-\frac{1}{2}+i \nu}(-\alpha, x ; b)=\left(\frac{2 b}{\pi}\right)^{1 / 2} \int_{1 / x}^{\infty} \tau^{\alpha-\frac{1}{2}} e^{-1 / \tau} K_{i \nu}(b \tau) d \tau .
$$

Integrating both sides in (56) with respect to $v$ from $v=0$ to $v=\infty$ using the fact ([47], page 153(6.5.13)) we get

$$
\begin{aligned}
\int_{0}^{\infty} \gamma_{-\frac{1}{2}+i v}(-\alpha, x ; b) d \nu & =\left(\frac{\pi b}{2}\right)^{1 / 2} \int_{1 / x}^{\infty} \tau^{\alpha-\frac{1}{2}} e^{-b \tau-\tau^{-1}} d \tau \\
& =\left(\frac{\pi}{2}\right)^{1 / 2} b^{-\alpha} \Gamma\left(\alpha+\frac{1}{2}, \frac{b}{x} ; b\right) .
\end{aligned}
$$


It should be noted that the process of integration under the integral sign in (57) is justified ([16], pages 427-448). Multiplying both sides in (57) by $\left(\frac{2}{\pi}\right)^{1 / 2} b^{\alpha}$ we get the proof of (53). The proof of (54) follows similarly. In particular substituting $\alpha=0$ in (53) and using (41) we get

$$
\begin{aligned}
& \int_{0}^{\infty} \gamma_{-\frac{1}{2}+i v}(0, x ; b) d v \\
& =\frac{\pi}{2 \sqrt{2}}\left[e^{-2 \sqrt{b}} \operatorname{Erfc}\left(\frac{1}{\sqrt{x}}-\sqrt{b x}\right)+e^{2 \sqrt{b}} \operatorname{Erfc}\left(\frac{1}{\sqrt{x}}+\sqrt{b x}\right)\right] .
\end{aligned}
$$

\section{Tabular and Graphical Representations}

For numerical and scientific computations, the extension functions $\gamma_{v}(\alpha, x ; b)$ and $\Gamma_{\nu}(\alpha, x ; b)$ can be tabulated by using IMSL FORTRAN subroutines for mathematical applications [39]. The values of the function can be calculated by using the numerical integration subroutine QDAGI. The subroutine uses a globally adaptive scheme in an attempt to reduce the absolute error. It should be noted that QDAGI is an implementation of the subroutine QAGI, which is fully documented by Piessens et al. [61]. The modified Bessel functions of the third kind (equations (8) - (9)) are computed by the IMSL subroutine BSKS which is based on the work of Cody [25]. On the other hand, subroutine GAMIC which is based on the computational procedure of Gautschi [32], is used for the incomplete Gamma function. It should be added that $\Gamma(\alpha, x ; 0)$, calculated by using the numerical integration QDAGI provides exactly the same results as that of the incomplete Gamma function calculated by the subroutine GAMIC.

The normalized representation of the function $\Gamma_{\nu}(\alpha, x ; b)$ for $v=0$ and various values of the parameters $\alpha$ and $b$ is given in [22]. The representation for $\nu \neq 0$ can be found similarly.

\section{Acknowledgement.}

The authors are indebted to the King Fahd University of Petroleum and Minerals for this research project. The useful comments made by the reviewer are appreciated.

\section{References}

[1] M. Abramowitz et al., "Handbook of Mathematical Functions", Nat. Bur. Std., Appl. Math. Ser. 55, U.S. Govt. Printing Office, Washington, D.C., (1964). 
[2] D. E. Amos, "Computation of modified Bessel functions and their ratios", Math. Comp. 17 (1974) 239-251.

[3] E. Artin, The Gamma Function, (Holt, New York, 1964).

[4] R. A. Askey, "The $q$-Gamma and $q$-Beta functions", Applic. Anal. 8 (1978) 125-141.

[5] R. A. Askey, "Ramanujan's extension of the Gamma and Beta functions", Amer. Math. Monthly 87 (1980) 346-359.

[6] A. C. Atkinson, "The simulation of generalized inverse Gaussian, generalized hyperbolic, gamma and related random variables", Research Report No. 52 (Dept. Theor. Statist., Aarhus University, 1979).

[7] A. K. Banerjee and G. K. Bhattacharyya, "Baysian results for the inverse Gaussian distribution with an application", Technometrics 21 (1979) 247-252.

[8] O. Barndorff-Nielsen, "Exponentially decreasing log-size distributions", Proc. R. Soc. London, A 353 (1977) 401-419.

[9] O. Barndorff-Nielsen, Information and exponential families in statistical theory (Wiley, Chichester, 1978).

[10] O. Barndorff-Nielsen, "Hyperbolic distributions and distributions on hyperbolae", Scand.J. Statist. 5 (1978) 151-157.

[11] O. Barndorff-Nielsen, P. Blaesild and C. Halgreen, "First hitting time models for the generalized inverse Gaussian distribution", Stoc. Processes Appl. 7 (1978) 49-54.

[12] O. Barndorff-Nielsen and D. R. Cox, "Edgeworth and saddle-point approximations with statistical applications", J.R. Statist. Soc. B 41 (1979) 279-312.

[13] O. Barndorff-Nielsen and C. Halgreen, "Infinite divisibility of the hyperbolic and generalized inverse Gaussian distributions", Z. Wahrscheinlichkeitstheorie Verw. Gebiete, 38 (1977) 309-311.

[14] P. Blaesild, "The shape of the generalized inverse Gaussian and hyperbolic distributions", Research Report No. 37 (Dept. Theor. Statist., Aarhus University, 1978).

[15] P. E. Böhmer, Differenzengleichungen und bestimmte Integrale (K. F. Koehler, Leipzig, 1939).

[16] B. M. Budak and S. V. Fomin, Multiple Integrals, Field Theory and Series, (Mir Publishers, 1978).

[17] B. C. Carlson, "A connection between elementary functions and higher transcendental functions", SIAM J. Appl. Math. 17 (1969) 116-148.

[18] H. S. Carslaw and J. C. Jaeger, Conduction of Heat in Solids, (Oxford University Press, 1959).

[19] M. Aslam Chaudhry and M. Ahmed, "On a probability function useful in size modeling", Canadian Journal of Forest Research, 23 (8) (1993) 1679-1683.

[20] M. Aslam Chaudhry and M. Ahmed, "On a class of probability functions - A unified approach" (in progress).

[21] M. Aslam Chaudhry and S. M. Zubair, "Analytic study of temperature solutions due to Gamma type moving point-heat sources", International Journal of Heat and Mass Transfer, 36 (6) (1991) 1633-1637.

[22] M. Aslam Chaudhry and S. M. Zubair, "Generalized incomplete Gamma functions with applications", Journal of Computational and Applied Mathematics (1993) (accepted for publication).

[23] R. S. Chhikara and J. L. Folks, "The inverse Gaussian distribution as a lifetime model", Technometrics 19 (1977) 461-468.

[24] R. S. Chhikara and J. L. Folks, "The inverse Gaussian distribution and its statistical application a review. (With discussion)", J. R. Statist. Soc. B 40 (1978) 263-289.

[25] W. J. Cody, "Algorithm 597: A sequence of modified Bessel functions of the first kind," $A C M$ Transactions on Mathematical Software 9 (1983) 242-245.

[26] D. R. Cox and P. A. W. Lewis, The statistical analysis of series of events (Methuen, London, 1966).

[27] A. Erdélyi et al,, Higher Transcendental Functions, Volume II, (McGraw-Hill, New York, 1953).

[28] A. Erdélyi et al., Tables of Integral Transforms, Volume I, (McGraw-Hill, New York, 1954).

[29] A. Erdélyi et al., Tables of Integral Transforms, Volume II, (McGraw-Hill, New York, 1954). 
[30] W. Feller, An Introduction to Probability Theory and Its Applications, Volume I, third ed. (1968); Volume 2, second ed. (1971) (Wiley, New York).

[31] R. A. Fisher, Statistical methods and scientific inference (Oliver and Boyd, Edinburgh, 1956).

[32] W. Gautschi, "A computational procedure for the incomplete Gamma function", ACM Transactions on Mathematical Software 5 (1979) 466-481.

[33] I. J. Good, "The population frequencies of species and the estimation of population parameters", Biometrika 40 (1953) 237-60.

[34] I. S. Gradshteyn and I .M. Ryzhik, Tables of Integrals, Series, and Products (Academic Press, New York, 1980).

[35] U. Grigull and H. Sandner, Heat Conduction, English Translation (ed. J. K. Kestin) (SpringerVerlag, Berlin, 1984).

[36] C. Halgreen, "Self-decomposability of the generalized inverse Gaussian and hyperbolic distributions", Z. Wahrscheinlichkeitstheorie Verw. Gebiete, 47 (1979) 13-18.

[37] H. Hochstadt, The Functions of Mathematical Physics (Wiley, New York, 1971).

[38] J. N. Hoem, "The statistical theory of demographic rates. A review of current developments. (With discussion)", Scand. J. Statist. 3 (1976) 169-185.

[39] IMSL MATH/LIBRARY Volume 2 (IMSL Inc., Houston, 1991).

[40] M. E. H. Ismail, "Integral representations and complete monotonicity of various quotients of Bessel functions”, Can. J. Math. 24 (1977) 1198-1207.

[41] M. E. H. Ismail and M. E. Muldoon, "Monotonicity of the zeros of a cross-product of Bessel functions", Siam. J. Math. Anal. 9 (1978) 759-767.

[42] H. Jeffreys and B. S. Jeffreys, Methods of Mathematical Physics, third ed. (Cambridge University Press, London, 1956).

[43] N. L. Johnson and S. Kotz, Distributions in statistics: continuous univariate distributions 1 (Houghton-Mifflin, Boston, 1970).

[44] B. Jorgensen, Statistical Properties of Generalized Inverse Gaussian Distributions, Lecture Notes in Statistics, Volume 9 (Springer-Verlag, New York, 1982).

[45] B. Jorgensen and B. V. Pedersen, "Contribution to the discussion of O. Barndorff-Nielsen and D. R. Cox: Edgeworth and saddle-point approximations with statistical applications", J. R. Statist. Soc. B 41 (1979) 309-310.

[46] N. Keyfitz, Introduction to the mathematics of population (Addison-Wesley, Reading, 1968).

[47] N. N. Lebedev, Special Functions and Their Applications, English Translation (ed. R. A. Silverman) (Prentice-Hall, New York, 1965).

[48] A. M. Legendre, Exercises de calcul intégral (Paris, 1811).

[49] A. M. Legendre, "Mémoire sur les integrations par arcs d'ellipse.", Histoire Acad. Roy. Sci. Avec Mém. Math. Phys. (1786) 616-643.

[50] F. Lombard, "A sequential test for the mean of an inverse Gaussian distribution", South African Statist. J. 12 (1978) 107-115.

[51] W. Magnus, F. Oberhettinger, and R. P. Soni, Formulas and Theorems for Special Functions of Mathematical Physics, third ed. (Springer-Verlag, New York, 1966).

[52] S. G. Mikhlin, Integral Equations and Their Applications to Certain Problems in Mechanics, Mathematical Physics and Technology (Pergamon Press, London, 1957).

[53] C. K. Mura, "Tests for the mean of the inverse Gaussian distribution", Scand. J. Statist. 5 (1978) 200-204.

[54] A. Nadas, "Best tests for zero drift based on first passage times in Brownian motion", Technometrics 15 (1973) 125-132.

[55] N. Nielsen, Theorie des Integrallogarithmus und verwandter Transcendenten (B. G. Teubner, Leipzig, 1906).

[56] N. Nielsen, Handbuch der Theorie der Gammafunktion (B. G. Teubner, Leipzig, 1906). 
[57] W. Nowacki, Thermoelasticity, second ed. (Pergamon Press, London, 1986).

[58] W. J. Padgett and L. J. Wei, "Estimation for the three-parameter inverse Gaussian distribution", Commun. Statist. - Theor. Meth. A 8 (1979) 129-137.

[59] S. A. Patil and J. L. Kovner, "On the power of an optimum test for the mean of the inverse Gaussian distribution", Technometrics 21 (1979) 379-381.

[60] J. R. Philip, “The function inv erf $\theta$ ", Austral. J. Phys. 13 (1960) 13-20.

[61] R. E. Piessens et al., QUAD-PACK (Springer-Verlag, New York, 1983).

[62] T. Roznowski, Moving Heat Sources in Thermoelasticity, English Translation (ed. R. T. Fenner) (Wiley, New York, 1989).

[63] A. L. Rukhin, "Strongly symmetric families and statistical analysis of their parameters", Zap. Naucvn. Sem. Leningrad. Otdel. Mat. Inst. Steklov 43 (1974), English Translation J. Soviet Math. 9 (1978) 886-910.

[64] E. Schrödinger, "Zur Theorie der Fall-und Steigversuche an Teilchen mit Brownscher Bewegung", Physikalische Zeitschrift 16 (1915) 289-295.

[65] V. Seshadri and J. J. Shuster, "Exact tests for zero drift based on first passage times in Brownian motion", Technometrics 16 (1974) 133-134.

[66] J. J. Schuster, "On the inverse Gaussian distribution function", J. Amer. Statist. Ass. 63 (1968) 1514-1516.

[67] H. S. Sichel, "On a distribution representing sentence-length in written prose", J. R. Statist. Soc. A 137 (1974) 25-34.

[68] H. S. Sichel, "On a distribution law for word frequencies”, J. Amer. Statist. Ass. 70 (1975) 542-547.

[69] B. Spain and M. G. Smith, Functions of Mathematical Physics (Van Nostrand-Reinhold, Princeton, 1970).

[70] D. A. Sprott, "Normal likelihoods and their relation to large sample theory of estimation", Biometrika 60 (1973) 457-465.

[71] M. C. K. Tweedie, "Statistical properties of inverse Gaussian distributions I", Ann. Math. Statist. 28 (1957) 362-377.

[72] G. A. Whitmore and M. Yalovsky, “A normalizing logarithmic transformation for inverse Gaussian random variables", Technometrics 20 (1978) 207-208.

[73] G. A. Whitmore, “An inverse Gaussian model for labour turnover”, J. R. Statist. Soc. A 142 (1979) $468-478$.

[74] M. G. Wise, "Skew distributions in biomedicine including some with negative powers of time." in Model building and Model selection, Dordrecht Statistical Distributions in Scientific Work, Volume 2 (eds. G. P. Patil et al.) (Dordrecht, Reidel, 1975) 241-262.

[75] S. M. Zubair and M. Aslam Chaudhry, "Temperature solutions due to continuously operating spherical-surface-heat sources in an infinite medium", Intl. Communications in Heat and Mass Transfer 18 (1991) 805-812.

[76] S. M. Zubair and M. Aslam Chaudhry, "Heat conduction in a semi-infinite solid subject to timedependent surface heat flux: an analytic study", Wärme-und Stoffübertragung 28 (1993) 357-364.

[77] S. M. Zubair and M. Aslam Chaudhry, "Temperature solutions due to continuously operating, Gamma-type heat sources in an infinite medium", ASME-HTD 207 (1992) 63-68. 